\title{
RADAR DE ONDA SUPERFICIAL DE HF (HFSWR)
}

\author{
Ariel Leiva L. ${ }^{1} \quad$ Guillermo Fernández S. ${ }^{1} \quad$ Raimundo Villarroel V. $^{1} \quad$ Emilio Quezada V. ${ }^{1}$ \\ Recibido el 8 de marzo de 2005, aceptado el 19 de agosto de 2005
}

\begin{abstract}
RESUMEN
Este trabajo pretende presentar las características generales más importantes de un sistema de vigilancia que usa Radar de Onda Superficial de HF (HFSWR). Actualmente, la Escuela de Ingeniería Eléctrica de la Universidad Católica de Valparaíso, en conjunto con la Armada de Chile, se encuentran comenzando los estudios acerca de esta tecnología para plantear variantes acerca de la misma. Los primeros estudios, que recién comienzan, tratan sobre el arreglo de elementos de recepción y los algoritmos de conformación digital de haz ("beamforming"). Este trabajo abordará, además, resultados de estos estudios.
\end{abstract}

Palabras clave: HFSWR, Onda Superficial, Beamforming.

\section{ABSTRACT}

This work presents the most important characteristics of a system of surveillance based on High Frequency Surface Wave Radar (HFSWR). Nowadays, the School of Electrical Engineering (Universidad Catolica de Valparaiso, Chile) together with the Chilean Navy, are beginning to study the HFSWR technology in order to establish the feasibility of its application in the coast of Chile. The first stage of this study, which recently had began, focuses on the arrangement of the receiving system elements and the algorithms of digital conformation of the receiving beam (beamforming).

Keywords: HFSWR, Surface Wave, Beamforming.

\section{INTRODUCCIÓN}

Chile posee un extenso territorio costero, por lo que le es imprescindible vigilar la actividad marítima de su Zona Económica Exclusiva (ZEE). La ZEE corresponde a las 200 MN (millas náuticas) desde la costa al mar abierto. Esta distancia supera ampliamente la curvatura de la Tierra (horizonte) [1], [2].

Según la Convención de la ONU con relación a los Derechos del Mar de 1992, los países tienen pleno derechos de explotación dentro de sus respectivas ZEE. Además de estos beneficios, los países tienen responsabilidades, como lo son la prevención de accidentes, contrabandos, contaminación, explotación ilegal y rescate de embarcaciones, entre otros [1], [2].

Debido a que las $200 \mathrm{MN}$ superan la distancia al horizonte desde la costa, cada país debe contar con sistemas de vigilancia efectivos distintos a los tradicionales.
En Chile, el Control de Tráfico Marítimo se basa en la recepción del posicionamiento de las embarcaciones que se encuentran dentro de las aguas nacionales de dos formas: Mediante el envío de su posición diaria hacia las Radioestaciones Marítimas, en el caso de las naves mercantes, y mediante la emisión desde un "posicionador" satelital que tienen a bordo en forma obligatoria para el caso de embarcaciones pesqueras industriales chilenas. En ambos casos, se trata de un sistema pasivo, en el cual se debe esperar que las embarcaciones se reporten diariamente. A su vez, hay embarcaciones nacionales y extranjeras de las cuales no se recibe posición y que podrían estar involucradas en situaciones de peligro, desde contrabando y pesca ilegal (con el alto costo en pérdidas para el país y la subsistencia de pescadores artesanales) o hasta amenazas a la seguridad nacional.

La alternativa más adecuada para una vigilancia en tiempo real de la ZEE sería contar con un sistema en que los sensores sean del tipo RADAR de Onda Superficial de

1 Pontificia Universidad Católica de Valparaíso, Av. Brasil 2147 Valparaíso, Chile, gfernand@ucv.cl 
HF (HFSWR), aprovechándose de las características de propagación de ondas de HF a través de la superficie del mar que les permite alcanzar distancias más allá del horizonte.

Una propuesta de este tipo de sensores ya se encuentra en uso en la costa este de Canadá, el cual fue desarrollado por Raytheon Systems Canada Ltd., en conjunto con el Departamento de Defensa Nacional de Canadá [2], [3].

El propósito de este trabajo es exponer las principales características de este tipo de sensores (HFSWR) en relación a la propagación de ondas superficiales de HF, estructura de un HFSWR, ruido e interferencia, reflectividad de señales de $\mathrm{HF}$, sistema de antenas transmisoras y receptoras, y otros. Cabe mencionar que la Escuela de Ingeniería Eléctrica de la PUCV en conjunto con la Armada de Chile, se encuentran comenzando los estudios pertinentes para proponer variantes a este tipo de sensores. Es por esta razón que se presentarán avances de resultados en relación al arreglo de antenas receptoras y algoritmos de conformación de haces digital ("beamforming") aplicado a HFSWR.

\section{PROPAGACIÓN DE SEÑALES EN LA BANDA HF}

La superficie, la tropósfera y la ionósfera son las responsables que el modelo ideal de propagación en espacio libre no sea correcto en la mayoría de los casos. La morfología y la conductividad de la superficie afectan la propagación. Además, la banda de frecuencia de trabajo va a definir los mecanismos de propagación de ondas que hay que considerar a la hora de analizar un enlace de radio.

Entre los mecanismos de propagación se cuentan: Propagación por Onda de Superficie, por Onda Ionosférica y por Onda Directa.

La propagación por Onda Superficial se produce en las frecuencias de señales de $\mathrm{HF}(3-30 \mathrm{MHz})$ hacia abajo. Esta onda se propaga en la discontinuidad tierra-aire. Este modo sólo propaga la polarización vertical, ya que la componente horizontal se atenúa muy rápidamente. El alcance que se obtiene varía ampliamente con la frecuencia y el tipo de suelo. Es de fácil análisis y comprensión cuando la superficie del mar se considera lisa, pero la realidad dista mucho de ésto, ya que es rugosa. Es por esta razón que se deben tener en cuenta los estados del mar cuando se desea predecir la atenuación. Cabe destacar que la atenuación es proporcional a la frecuencia y a los estados del mar. El campo radiado puede expresarse como una suma de términos, cuyas amplitudes son función de la frecuencia, el tipo de terreno, la altura de las antenas sobre el suelo, la polarización y la distancia. Cabe destacar que la intensidad de campo disminuye con la frecuencia, de forma que la propagación por onda superficial deja de ser un mecanismo de propagación a grandes distancias en frecuencias altas. La atenuación disminuye al aumentar la conductividad del terreno, así sobre el agua de mar el comportamiento del campo es prácticamente $1 / \mathrm{R}$, mientras que para tierras secas es $1 / R^{2}$. Un caso a considerar es el tramo desde la antena hasta la discontinuidad tierra-mar, en que la propagación es por tierra, por lo tanto, las antenas deben estar situadas lo más cercanas posible al mar con motivo de disminuir las pérdidas por propagación por tierra.

En cuanto a la Ionósfera, ésta es la región de las capas altas de la atmósfera (60 a $400 \mathrm{~km}$ de altura) que debido a su ionización refleja las señales radioeléctricas hasta aproximadamente los $30 \mathrm{MHz}$. La ionización, o presencia de electrones libres, se produce fundamentalmente por las radiaciones solares en las bandas ultravioletas y de rayos $\mathrm{X}$, por los rayos cósmicos y por meteoritos. Esto hace que la densidad de electrones varíe según la hora del día, estación del año y otros. Además, varía con la altura. En las bandas MF y HF, principalmente, la Ionósfera refracta las ondas radioeléctricas, haciendo que éstas retornen a la tierra. Este mecanismo se denomina refracción ionosférica. La distancia de los saltos dependerá de la frecuencia, del ángulo de incidencia, hora del día, etc.

Para frecuencias superiores a VHF, el mecanismo de propagación es el de Onda Directa. En estas frecuencias, la Ionósfera se hace transparente y los mecanismos de propagación se ven afectados por la influencia del suelo (reflexiones y difracciones) y por la Tropósfera (mediante refracción, atenuación y esparcimiento). Debido a que este mecanismo prevalece a frecuencias superiores a las HF no se continuará con su análisis.

\section{SISTEMA BÁSICO DE HFSWR}

El HFSWR explota la propagación de señales de HF por la superficie del mar: transmite señales en esta banda por onda superficial más allá del horizonte (ver figura 1), las que se reflejan (eco) y vuelven al receptor. Cabe recordar que sólo la componente vertical podrá propagarse como onda superficial.

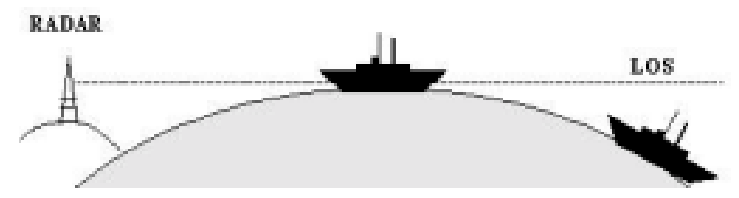

Fig. 1 Esquema de alcance de un HFSWR. 
Un sistema de vigilancia que usa este tipo de RADAR se compone, generalmente, de lo siguiente [2]:

- Sistema de Vigilancia o sensores HFSWR.

- Sistema de Identificación Directa.

- Sistema de Identificación Indirecta.

- Sistema que fusione y controle la información entregada por los anteriores sistemas.

Algunas características de un HFSWR son:

- Opera en el modo pulso-doppler.

- La zona de vigilancia es iluminada por un arreglo de antenas transmisoras direccional (120 ${ }^{\circ}$ ancho del haz).

- Ecos, desde todos los objetos dentro del área de cobertura, son recibidos por un arreglo lineal de antenas.

- Técnicas de procesamiento digital de señales son usadas en el arreglo receptor para conformar el haz ("beamforming" o filtraje espacial) y el movimiento o "barrido" de éste es a través de $120^{\circ}$ de azimut con un ancho del haz de 5 a $8^{\circ}$.

- El eco retornado es analizado de acuerdo al rango, velocidad y orientación.

- Las detecciones son comparadas con un umbral mediante la técnica de CFAR ("Constant False Alarm Rate"). Las detecciones que exceden el umbral son declaradas como detecciones válidas.

La figura 2 muestra un esquema físico de este tipo de sensores:

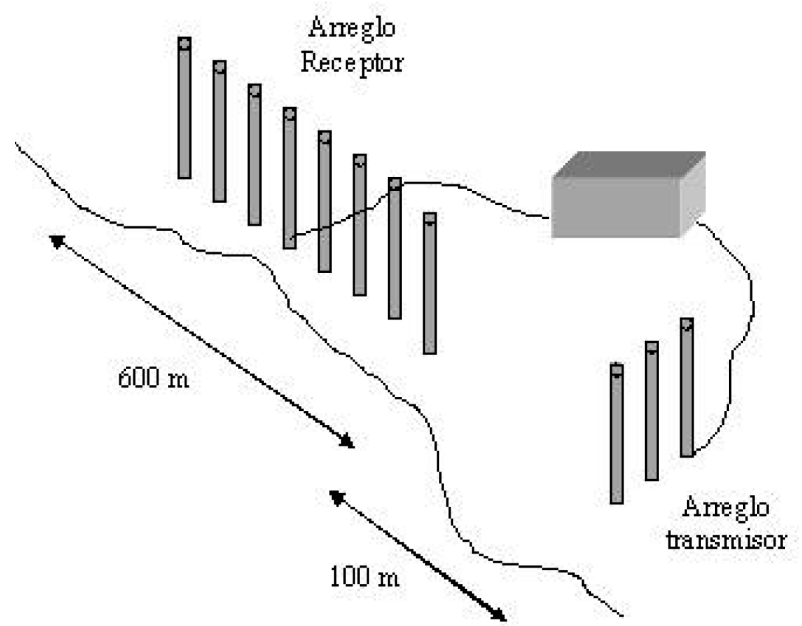

Fig. 2 Esquema básico y típico de un HFSWR.

\section{ASPECTOS FÍSICOS RELEVANTES}

La física del HFSWR varía en comparación con los de microondas. Algunos aspectos físicos importantes a considerar en HFSWR son:

- Características de propagación de ondas superficiales sobre tierra esférica con superficie rugosas (olas) y posibles islas.

- Comportamiento del RCS ("RADAR Cross Section" o Sección Transversal de RADAR) del objetivo, el cual tiene dimensiones comparables a $\lambda$ de operación.

- Predecir el comportamiento de grandes y largos arreglos de antenas transmisoras y receptoras sobre suelos con pérdidas.

- Detectar una señal objetivo inmersa en ruido, interferencia y "clutter" (retorno) marino.

\section{Ecuación HFSWR}

El HWSR tiene una excelente capacidad para detectar, a gran distancia, barcos y icebergs, por ejemplo. La potencia recibida desde un objetivo para un HFSWR "monoestático" es definido como [2]:

$$
P_{r}=\frac{P_{t} G_{t} G_{r} \sigma A^{4} \lambda^{2}}{(4 \pi)^{3} R^{4} L_{s}}
$$

donde:

$P_{r}$ es la potencia peak recibida.

$P_{t}^{r}$ es la potencia peak transmitida.

$G_{t}$ es la ganancia de la antena transmisora.

$G_{r}$ es la ganancia de antena receptora.

$\sigma$ es el "Radar Cross Section" (RCS) del objetivo.

$\lambda$ longitud de onda de operación del radar.

$\mathrm{R}$ es el rango del objetivo.

$\mathrm{L}_{\mathrm{S}}$ son las pérdidas del sistema.

$A$ es el factor de atenuación de Onda Superficial (SW) de Norton a un rango $\mathrm{R}(\mathrm{A}<1)$.

\section{Ruido}

Los niveles de Ruido se deben, principalmente, a tres contribuciones [2]:

- Ruido Externo.

- Autointerferencia Ionosférica.

- "Sea Clutter" o retorno del mar. 


\section{Ruido Externo}

Se debe principalmente a las señales provenientes de lugares lejanos que usan comunicación vía onda ionosférica o "sky" que, debido a las características, cambiantes de las capas de la Ionósfera, pueden llegar a las instalaciones del RADAR. Este tipo de ruido es predicho por la ITU. Tiene características que difieren del día y la noche debido a la desaparición de la capa $\mathrm{E}$. Además, este ruido es mayor a más baja frecuencia.

\section{Autointerferencia Ionosférica}

Una de las causantes más importantes de interferencia en HFSWR es la Ionósfera.

En la figura 3 se ilustran posibles caminos de interferencia, mostrando los retardos ya sea para la onda "sky" como para la onda superficial. Estos retardos se traducen en ruido no deseado en el sistema. Estos retardos varían según la hora debido a las características cambiantes de las capas de la Ionósfera. Estos trayectos interferentes varían en el tiempo debido a las características cambiantes de las capas de la Ionósfera. Para mitigar este efecto dañino, es deseable que los sistemas transmisores y receptores cuenten con nulos en sus lóbulos de radiación para señales provenientes o hacia direcciones cercanas a la vertical.

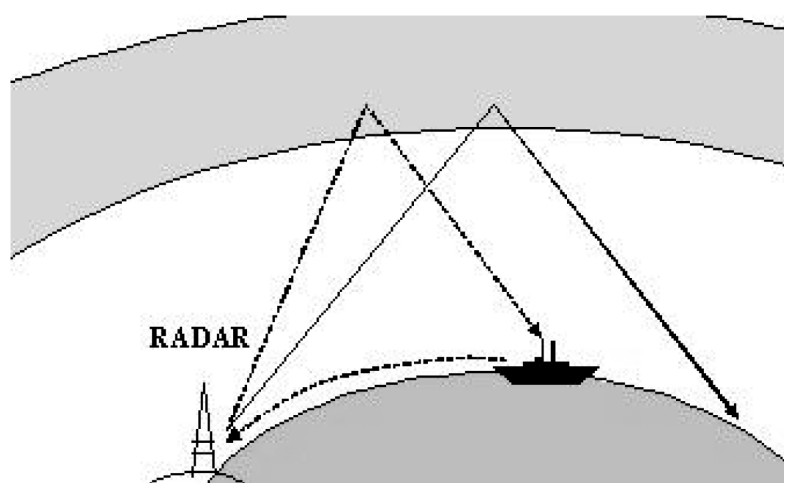

Fig. 3 Distintas señales de retorno debido a la propagación ionosférica.

Otra medida, además, sería que el RADAR use la frecuencia crítica (CF) para el día, donde las ondas "sky" son absorbidas por la capa $\mathrm{D}$. Alternativamente, se podría usar una frecuencia cercana a la máxima utilizable (MUF) en la que la energía propagada por onda "sky" a través de la ionósfera no es devuelta a la Tierra.

\section{"Sea Clutter"}

El eco retornado desde el "clutter" (desorden) de la superficie oceánica (olas) tiene características de espectro
Doppler. La contribución dominante es producida por el esparcimiento desde las ondas oceánicas que tienen una $\lambda$ correspondiente a la mitad de la del RADAR, moviéndose radialmente hacia o fuera del dispositivo. De aquí se desprenden dos tipos de frecuencias Doppler de retorno [2]:

$$
f_{D}= \pm \sqrt{\frac{g}{\pi \lambda}}[H z]
$$

correspondientes a las velocidades de propagación de esas olas. Donde "g" es la aceleración de gravedad y " $\lambda$ " es la longitud de onda del RADAR. Este fenómeno dificulta la detección de embarcaciones a baja velocidad si el mar se encuentra muy agitado.

\section{Reflectividad o RCS de los objetivos}

La reflectividad de los objetivos es diferente al caso de la banda de microondas, mientras que en este último es de régimen óptico, en HFSWR nos encontramos en el régimen de Resonancia ( $\lambda$ comparables a las dimensiones de los objetivos).

También cabe destacar que RCS varía de acuerdo al ángulo de incidencia, frecuencia y tamaño. Además, de la interacción mutua entre objetivos que se encuentran cercanos entre sí.

\section{Espectro Saturado}

En la elección de la frecuencia de operación del HFSWR se deben tener en cuenta las fuentes de interferencia. Se debe tratar de encontrar alguna pequeña banda desocupada en un espectro saturado. De acuerdo al plan de frecuencias entregado por la Subtel, este espectro se encuentra completamente saturado, por lo que se debe plantear un proyecto a la misma organización para solicitar la desocupación de alguna banda de radiofrecuencia de HF.

\section{REQUERIMIENTOS DE LOS SISTEMAS DE ANTENAS}

\section{Suelo}

Las antenas siempre están ubicadas en un suelo susceptible a las pérdidas. Los parámetros eléctricos del suelo juegan un rol crítico en el desempeño del arreglo de antenas.

En HF, la superficie del océano actúa como conductor eléctrico perfecto (PEC). Una superficie PEC tiene una 
impedancia ideal a $0 \Omega$. Un elemento vertical de $1 / 4$ sobre una superficie PEC actúa como un elemento de $1 / 2$ en espacio libre. Es por esta razón que el diagrama de radiación tiene su máximo en el plano horizontal. Ésta es la meta del diseño para un sistema de antena de HFSWR.

Un suelo con parámetros $\varepsilon_{\mathrm{g}}$ y $\sigma_{\mathrm{g}}$ tiene una impedancia de superficie dada por:

$$
Z_{s}=120 \pi\left[\frac{i \omega \varepsilon_{o}}{\sigma_{g}+i \omega \varepsilon_{g}}\right]^{1 / 2}\left[1+\frac{i \omega \varepsilon_{o}}{\sigma_{g}+i \omega \varepsilon_{g}}\right]^{1 / 2}
$$

Para poder tener un nivel de impedancia de superficie aceptable se puede realizar, básicamente, de dos maneras:

- El arreglo de antenas puede estar lo más cercano a la orilla del mar.

- Usar sistemas de alambres (radiales u otros) en cada una de las antenas para mejorar la conductividad del terreno.

\section{Arreglo de Antenas}

Ya se revisaron conceptos sobre la localización física de los arreglos de antenas.

Además, la localización de éstas debe ser lo más simétrica posible para que todas las pérdidas sean las mismas, y no deben incluir grandes bordes rocosos muy pronunciados.

\section{Arreglo Transmisor}

El arreglo transmisor debe tener una gran ganancia en la banda de HF a usar. Además, tiene que poseer un ancho del haz de $120^{\circ}$ para cubrir la zona de vigilancia con polarización vertical para la propagación superficial. Del mismo modo, deberá tener un buen plano de tierra para mejorar las impedancias y disminuir la radiación hacia la ionósfera.

Generalmente, se usan antenas semilogarítmicas periódicas con polarización vertical para este objetivo.

\section{Arreglo Receptor}

Como se presentarán más adelante resultados parciales acerca de los arreglos de antenas receptoras y algoritmos de conformación de haces digital, se detallarán aspectos relevantes sobre las características del arreglo receptor de un HFSWR.
Deberá ser un arreglo de varias antenas, en alguna configuración en especial, que cumplan los siguientes requerimientos:

- Poseer una polarización vertical.

- Ancho del haz muy angosto (a mayor número de antenas en el arreglo es posible formar haces más angostos, pero con el aumento del terreno a utilizar en la instalación de las antenas).

- Gran relación frente-espalda.

- Direccionamiento simple del haz (barrido) mediante técnicas de "beamforming" digital. Para implementar los algoritmos, se debe, primeramente, diseñar el arreglo de antenas. Un sistema de "beamformer" es aquel que tenga la capacidad de escuchar señales muy débiles que se encuentren a una gran distancia y con una dirección de "escucha" controlable.

- Se debe instalar el arreglo muy cercano al mar y paralelo a la línea costera para mejorar las pérdidas debido a la interacción con suelos pobres.

- Poseer un buen plano de tierra (con radiales u otros) para minimizar la radiación en otras direcciones que no sean del plano horizontal.

- Fácil implementación de técnicas de supresión de radiación en ciertas direcciones interferentes (filtraje espacial auxiliar).

\section{PROPUESTAS DE ARREGLOS DE ANTENAS Y "BEAMFORMING" DIGITAL PARA EL SISTEMA RECEPTOR}

Se presentarán, a continuación, conceptos relevantes, propuestas y resultados de simulaciones de diagramas de radiación para diferentes tipos de arreglos de antenas receptoras en relación a la configuración física del mismo $\mathrm{y}$ a diferentes algoritmos de conformación de haces.

\section{HPBW vs. Resolución}

En los sistemas de Radar, el factor más influyente sobre la resolución azimutal corresponde al ancho del lóbulo principal con que se detectarán los blancos. Es decir, que a un menor ancho del lóbulo, podremos distinguir mejor entre dos blancos muy cercanos, y a medida que el lóbulo aumenta su ancho estos dos blancos tenderán a confundirse en un solo blanco.

El ancho de lóbulo principal, también conocido como "Half-Power Beamwidth" (HPBW), es cuantizado en grados y se define como la apertura en grados entre los dos puntos del lóbulo principal que se encuentran a $-3 \mathrm{~dB}$ del máximo. 
En un arreglo de antenas como el de nuestro interés, existen dos variables que pueden modificar el ancho de un lóbulo principal. Estas son el número de elementos que componen el arreglo, y la separación entre estos elementos. Esta es la razón por la cual se le dará énfasis especial a estas dos variables en el momento de diseñar arreglo de antenas.

El HPBW para un arreglo lineal de "N" elementos y con separación "d" entre ellos está dado por [5]:

$$
\begin{aligned}
H P B W= & \cos ^{-1}\left[\cos \theta_{o}-0.443 \frac{\lambda}{(L+d)}\right]- \\
& \cos ^{-1}\left[\cos \theta_{o}+0.443 \frac{\lambda}{(L+d)}\right]
\end{aligned}
$$

Notar que $\mathrm{N}=(\mathrm{L}+\mathrm{d}) / \mathrm{d}$, y $\theta_{\mathrm{o}}$ es el ángulo hacia donde está dirigido el haz principal, por lo tanto, $\mathrm{N}$ está implícito en la ecuación anterior. L es el largo total del arreglo.

A continuación se analizarán por separado, vía simulación, las variables que influyen en el HPBW de la ecuación 4.

\section{HPBW vs. Separación entre Elementos}

La primera variable a tratar corresponde a la separación de los elementos (d) del arreglo. Se simularon 3 arreglos lineales de antenas de $24 \times 1$ elementos con diferentes espaciados de $0,25 \lambda, 0,51$ y $0,75 \lambda$, respectivamente. Los diagramas de radiación simulados se muestran en las figuras 4,5 y 6 . Cabe destacar que todos los elementos en este trabajo corresponden a monopolos de $0,25 \lambda$.

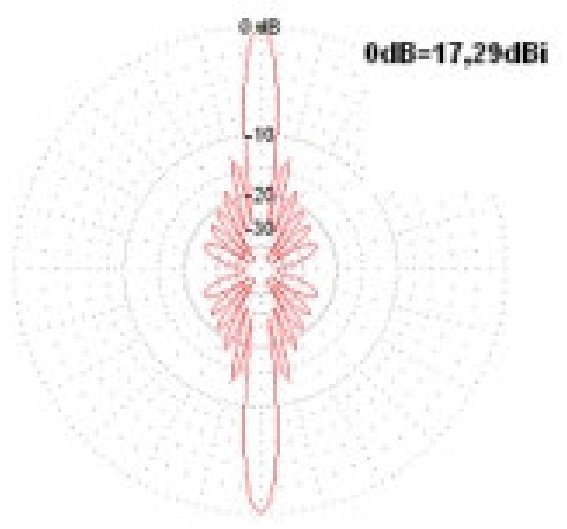

Fig. 4 Diagrama de radiación horizontal de un arreglo lineal $24 \times 1$ Broadside (monopolos de $0,25 \lambda$ ) separados $0,25 \lambda$. En este caso $0 \mathrm{~dB}=17,29 \mathrm{dBi}$.

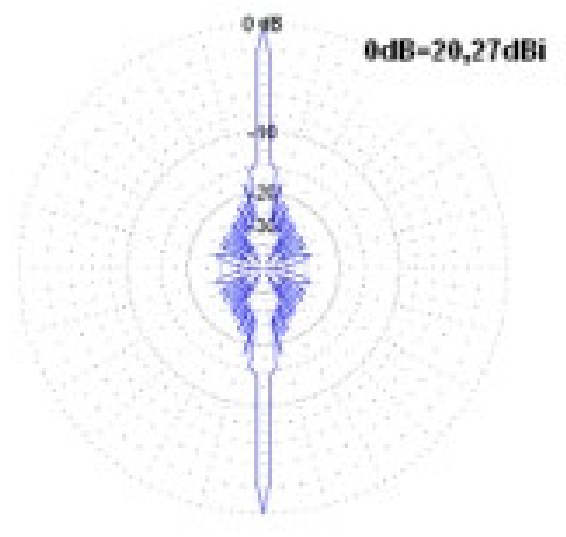

Fig. 5 Diagrama de radiación horizontal de un arreglo

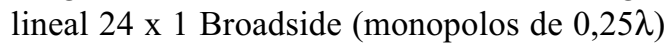
separados $0,5 \lambda$.

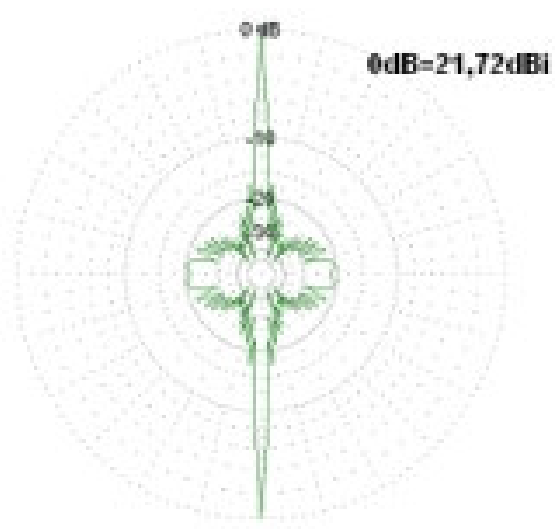

Fig. 6 Diagrama de radiación horizontal de un arreglo lineal 24 x 1 Broadside (monopolos de $0,25 \lambda$ ) separados $0,75 \lambda$.

La siguiente tabla muestra un resumen de los resultados obtenidos.

Tabla 1 HPBW y su relación con "d".

\begin{tabular}{|l|c|c|c|}
\hline & $\mathrm{d}=0,25 \lambda$ & $\mathrm{d}=0,5 \lambda$ & $\mathrm{d}=0,75 \lambda$ \\
\hline HPBW & $8.4^{\circ}$ & $4,2^{\circ}$ & $1,8^{\circ}$ \\
\hline $\begin{array}{l}\mathrm{N}^{\circ} \text { de lób. } \\
\text { sec. sobre } \\
\text { los }-15 \mathrm{~dB}\end{array}$ & 4 & 0 & 0 \\
\hline $\begin{array}{l}\text { Presencia de } \\
\text { lób. sec. con } \\
\text { gran área }\end{array}$ & $\mathrm{NO}$ & $\mathrm{NO}$ & $\mathrm{SI}$ \\
\hline $\begin{array}{l}\text { Ganancia } \\
\text { [dBi] }\end{array}$ & 17,29 & 20,27 & 21,72 \\
\hline
\end{tabular}


En el caso de un HPBW de $8,4^{\circ}(\mathrm{d}=0,25 \lambda)$ se presentan los arcos equivalentes del HPBW para distintos rangos:

Tabla 2 Arco vs. Rango (N=24).

\begin{tabular}{|c|c|}
\hline $\begin{array}{c}\text { HPBW }=8,4^{\circ} \\
\text { Rango }[\mathrm{Km}]\end{array}$ & $\begin{array}{c}\mathrm{d}=0,25 \lambda \\
\text { Arco }[\mathrm{Km}]\end{array}$ \\
\hline 370 & 54 \\
\hline 277 & 40 \\
\hline 185 & 27 \\
\hline 92 & 13 \\
\hline
\end{tabular}

Y para el caso de $H P B W=4,2^{\circ}(d=0,5 \lambda)$ :

Tabla 3 Arco vs. Rango (N=24)

\begin{tabular}{|c|c|}
\hline $\begin{array}{c}\text { HPBW }=4,2^{\circ} \\
\text { Rango }[\mathrm{Km}]\end{array}$ & $\begin{array}{c}\mathrm{d}=0,5 \lambda \\
\text { Arco }[\mathrm{Km}]\end{array}$ \\
\hline 370 & 27 \\
\hline 277 & 20 \\
\hline 185 & 13 \\
\hline 92 & 6.7 \\
\hline
\end{tabular}

Como podemos observar, el arco está linealmente asociado con el rango en el cual se encuentra nuestro blanco, es decir, que a mayor distancia tendremos menos capacidad para distinguir entre dos blancos.

Finalmente se puede ver con claridad que mientras más delgado sea el haz del RADAR mayor resolución se obtendrá.

Bajo este análisis queda claro que la mejor decisión sería la elección de un arreglo con separación de $0,5 \lambda$, ya que es muy superior en lo que se busca con respecto a un arreglo con separación de $0,25 \lambda$, ya sea en el nivel de lóbulos secundarios como en el ancho de lóbulo principal. Si bien un arreglo con separación $0,75 \lambda$ tiene un lóbulo principal bastante más angosto que un arreglo con separación de separación $0,5 \lambda$, debe ser descartado debido a la presencia de un lóbulo secundario de gran área que juega en contra del diseño al momento de mover el lóbulo principal a lo largo del área de azimut requerida en el RADAR.

\section{HPBW v/s Número de Elementos}

La segunda variable que tiene relación con el HPBW es el número de elementos que componen el arreglo lineal básico. Se realizaron tres simulaciones de un arreglo lineal con separaciones entre elementos de $0,5 \lambda$, y distintos números de elementos $(\mathrm{N})$ para apreciar la relación con el HPBW.

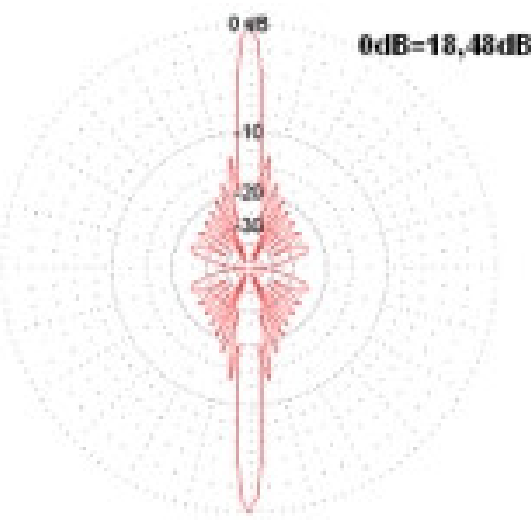

Fig. 7 Diagrama de radiación horizontal de un arreglo

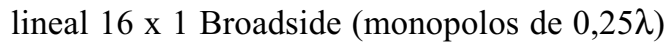
separados $0,5 \lambda$.



Fig. 8 Diagrama de radiación horizontal de un arreglo

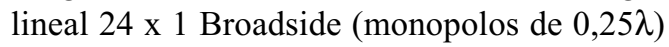
separados $0,5 \lambda$.

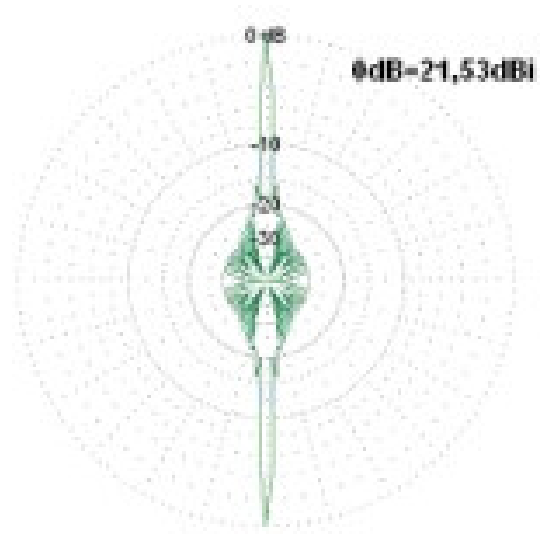

Fig. 9 Diagrama de radiación horizontal de un arreglo

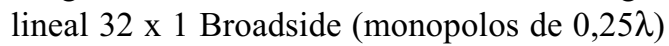
separados $0,5 \lambda$. 
A continuación, se presenta la tabla 4 que contiene los datos del ancho del haz de los distintos arreglos:

Tabla 4 HPBW y Ganancia v/s N.

\begin{tabular}{|c|c|c|c|}
\hline $\mathrm{N}$ & 16 & 24 & 32 \\
\hline HPBW & $6^{\circ}$ & $4,2^{\circ}$ & $2,4^{\circ}$ \\
\hline Ganancia[dBi] & 18,48 & 20,27 & 21,53 \\
\hline
\end{tabular}

Otra razón por la que se decide conformar el arreglo con 24 elementos o más, es que, a partir de los 24 elementos hacia arriba, el nivel de los lóbulos secundarios se ve fuertemente disminuido, quedando prácticamente todos bajo los $-20 \mathrm{~dB}$. Otro punto importante a considerar es la ganancia que se obtiene de los distintos arreglos lineales. Como podemos apreciar, la diferencia entre la ganancia del arreglo de 32 elementos y el de 24 corresponde a 1,24 $\mathrm{dBi}$, y la diferencia entre el de 24 y 16 corresponde a 1,79 $\mathrm{dBi}$. No son diferencias significativas, por lo tanto, no es un factor preponderante en el diseño.

\section{Supresión del segundo lóbulo principal de un arreglo lineal}

En arreglos lineales, lamentablemente, no existe un solo lóbulo principal, sino que existen dos del mismo tamaño $\mathrm{y}$ forma. Esto se puede apreciar en las figuras 4 a la 9.

Un RADAR requiere un solo lóbulo principal lo más angosto posible para evitar que perciba señales provenientes de otras direcciones a la deseada [4]. Es por esta razón, que se necesita suprimir o cancelar el segundo lóbulo principal. La supresión del segundo lóbulo puede lograrse principalmente con un arreglo bidimensional, además, de lograr otro requisito principal como es el aumentar la ganancia del arreglo.

En la práctica, esto se traduce en multiplicar el diagrama de radiación producido por el arreglo lineal (eje "X") con el diagrama de radiación producido por las antenas en el arreglo bidimensional (eje "Y") que en este caso son 2 antenas en modo "end-fire". La figura 10 muestra un diagrama de radiación para el eje "Y", el cual se multiplicará por el del arreglo lineal de la figura 8.

El pattern de la figura 10 resulta el ideal para cancelar el segundo lóbulo del arreglo lineal. Para entenderlo de otra manera en términos más prácticos, este pattern multiplica por 1 los lóbulos de los primeros dos cuadrantes de un arreglo lineal y por 0 los dos cuadrantes restantes. Sin embargo, esto no es del todo cierto, ya que en parte este pattern ocupa una pequeña fracción en los 2 cuadrantes posteriores, es decir, $3^{\text {er }}$ y $4^{\circ}$ cuadrantes. Dadas estas consideraciones, el arreglo propuesto consta de 24 elementos en el eje X y 2 elementos en el eje Y. Las separaciones entre elementos son de 0,51 y $0,25 \lambda$ en el eje $\mathrm{X}$ e $\mathrm{Y}$, respectivamente. El desfase entre elementos en $\mathrm{X}$ es $0^{\circ}$ y $90^{\circ}$ en $\mathrm{Y}$. Ver figura 11.

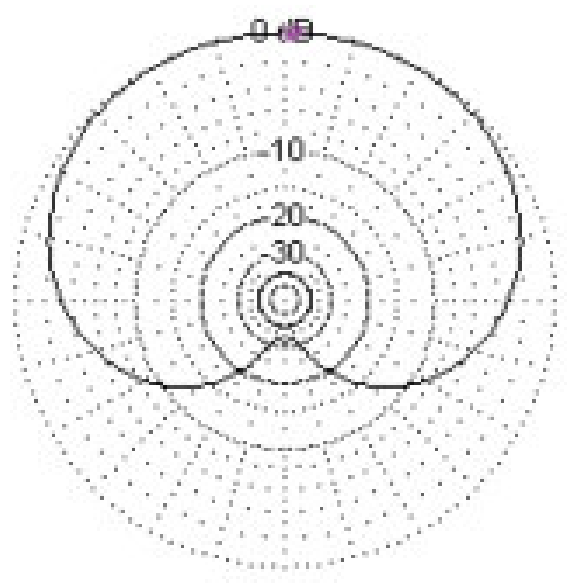

Fig. 10 Diagrama de radiación de 2 monopolos de $0,25 \lambda$ separados $0,25 \lambda$ y en cuadratura de fase.

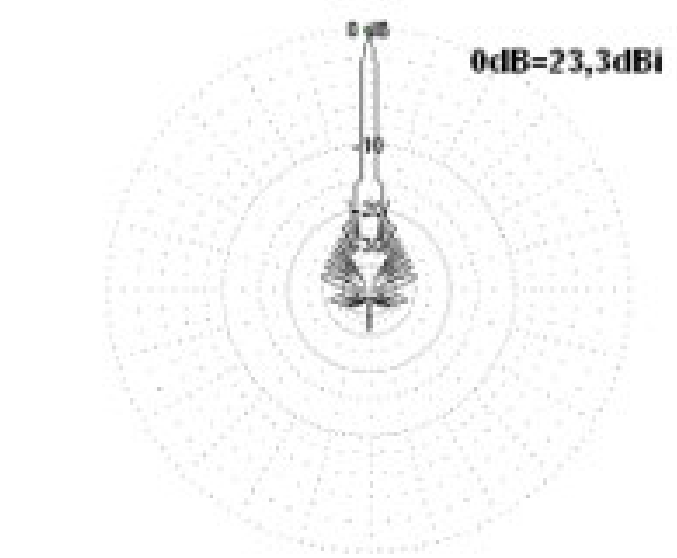

Fig. 11 Pattern de radiación de un arreglo bidimensional de $24 \times 2$ (monopolos de $0,25 \lambda$ ) con separación de $0,5 \lambda$ y $0,25 \lambda$ en $X$ e $Y$, respectivamente.

Cabe destacar que este beampattern debe cubrir o "barrer" $120^{\circ}$ de azimut. De esta forma, la figura 11 solo cumple perfectamente los requisitos para un arreglo "broadside". Recordemos que por el principio de multiplicación de patterns el arreglo lineal básico consta de 2 lóbulos principales de los cuales el lóbulo trasero es anulado por la configuración bidimensional que consta de la multiplicación con un pattern del tipo de la figura 10 . Pero cuando se necesita variar el ángulo del haz principal, el segundo haz tiende a juntarse con éste, por lo que el beampattern del arreglo bidimensional de la figura 10 no es capaz de anularlo completamente. En este caso, son necesarias técnicas de filtrado espacial ("beamforming" digital) para la supresión de lóbulos secundarios e interferencias. 
En las gráficas anteriormente mostradas, se logra apreciar la diferencia fundamental entre arreglos de antenas lineales y bidimensionales. La principal diferencia radica en que los arreglos lineales poseen al menos 2 lóbulos principales, mientras que los bidimensionales (si se escoge una adecuada separación) poseen solamente uno. Es por esta razón fundamental que se recomienda usar arreglos de antenas bidimensionales. El ejemplo mostrado en este informe cumple con las características requeridas para un HFSWR. Se obtuvieron anchos de lóbulos principales de $4,2^{\circ}$ y relación entre el lóbulo principal y secundario de un poco más de $15 \mathrm{~dB}$. Por las razones anteriormente expuestas, el arreglo bidimensional de $24 \times 2$ elementos es el elegido.

Cabe mencionar que aún nos encontramos con niveles de lóbulos secundarios bastante altos, pero hay que resaltar que éstos resultan de sólo la síntesis del "beampattern" propio del arreglo sin ningún procesamiento posterior de "beamforming" digital.

\section{Efectos del suelo en el diagrama de radiación}

Las gráficas anteriores mostraban el diagrama de radiación suponiendo un suelo eléctricamente perfecto. Lamentablemente, la realidad dista mucho de serlo.

El efecto de no tener una PEC será una disminución de la ganancia $\mathrm{y}$, además, de una elevación de la radiación en el plano vertical. El ángulo entre el plano horizontal y la máxima radiación en el plano vertical se denomina TOA "Take of Angle". Un monopolo vertical sobre una PEC tiene un TOA de $0^{\circ}$ y a medida que las pérdidas del suelo se incrementan el TOA también lo hará.

Una superficie con buenas características eléctricas será la que tiene una gran conductividad y permitividad. También sabemos que el océano actúa prácticamente como una PEC en HF. La ecuación 3 muestra las variables involucradas en la impedancia del terreno, en la que queda claro la dependencia con la frecuencia.

Para mejorar las características conductoras del suelo donde se instalará el arreglo de antenas se proponen dos soluciones: humedecer el terreno y/o instalar radiales.

Existen algunas diferencias fundamentales entre los diagramas de radiación (o recepción) entre un mismo arreglo de antenas para distintos tipos de suelos.

La figura 12 muestra el pattern de radiación vertical del arreglo escogido sobre una PEC.

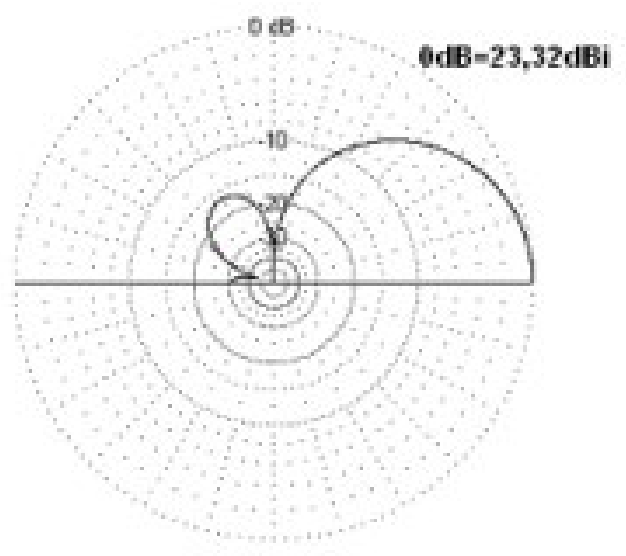

Fig. 12 Pattern de radiación vertical de un arreglo 24 × 2 sobre una PEC.

En este caso, la ganancia máxima corresponde a 23,32 $\mathrm{dBi}$ que se encuentra en el plano horizontal, o sea, TOA $=0^{\circ}$.

El siguiente caso muestra los pattern de radiación verticales para el mismo arreglo 24 × 2 sobre una tierra $\operatorname{seca}\left(\epsilon_{\mathrm{r}}=4, \mathrm{~s}=0,001 \Omega^{-1} / \mathrm{m}\right)$ y una húmeda $\left(\epsilon_{\mathrm{r}}=30 \sigma=0,02\right.$ $\left.\Omega^{-1} / \mathrm{m}\right)$. Ver figura 13 .

Es claro el mejor desempeño para un arreglo en tierra húmeda en comparación con la tierra seca que se aprecia en la ganancia. Lamentablemente, el TOA aumenta a $20^{\circ}$ al no tratarse de una PEC.

En relación a plano de elevación del diagrama de radiación, se aprecia claramente que la radiación comienza a separarse de la superficie horizontal cuando el suelo comienza a ser eléctricamente imperfecto. A más imperfecto el suelo, más radiación hacia el cielo se producirá. Esto produce un aumento en la Autointerferencia Ionosférica.

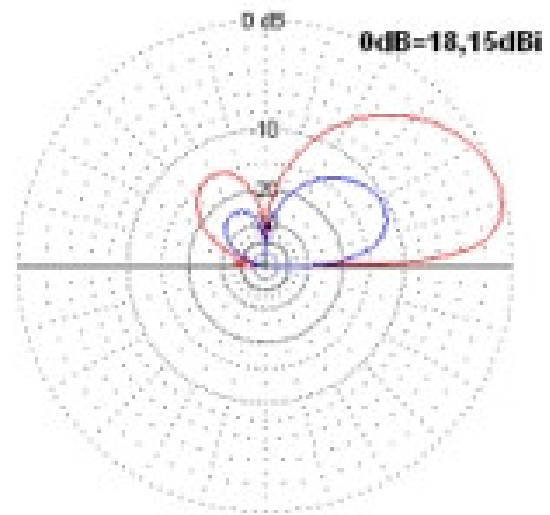

Fig. 13 Pattern de radiación vertical de un arreglo 24 x 2 sobre un suelo seco (azul) y sobre suelo húmedo (rojo). 
Cabe destacar que para este tipo de aplicación RADAR se necesita una máxima energía en el sentido horizontal $\mathrm{u}$ onda superficial por lo que este efecto, descrito anteriormente, es nocivo para las pretensiones del HFSWR.

Además, se simuló el efecto de la incorporación de radiales para mejorar la conductividad del terreno para así disminuir el TOA. Se simularon los diagramas de radiación verticales para el arreglo de $24 \times 2$ con 16 radiales cada uno (Figura 14).

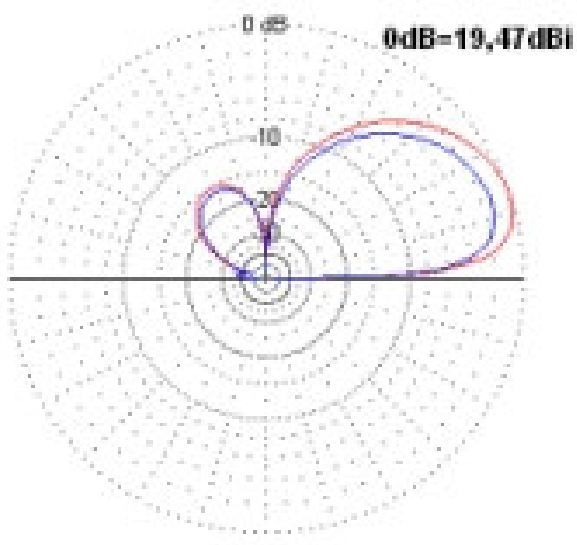

Fig. 14 Pattern de radiación vertical del arreglo $24 \times 2$ para tierra húmeda $\left(\epsilon_{\mathrm{r}}=30 \sigma=0,02 \Omega^{-1} / \mathrm{m}\right) \sin$ radiales (azul) y con radiales (rojo).

En relación al plano ecuatorial cabe destacar que los diagramas de radiación ensanchan su lóbulo principal a medida que el suelo se vuelve eléctricamente pobre o imperfecto. Esto provoca que se dificulte la detección de dos objetivos que se encuentren cercanos a una misma distancia del RADAR, pero a diferentes ángulos de acuerdo al azimut.

Por lo que se recomienda instalar radiales de material conductor para mejorar el plano de tierra del arreglo de antenas y/o instalar el arreglo lo más cercano a la superficie marina, ya que ésta tiene propiedades eléctricas muy buenas.

\section{Filtraje espacial para la cancelación de interferencias y lóbulos secundarios}

El arreglo de antenas bidimensional escogido de $24 \times 2$ cumple con los requisitos de una alta ganancia (sobre los $20 \mathrm{dBi}$ ), un HPBW angosto $\left(4,2^{\circ}\right)$ y una supresión parcial del segundo lóbulo principal. Es necesario, entonces, cancelar las interferencias provenientes de direcciones distintas a las del lóbulo principal. Entre las principales interferencias se encuentran las señales de comunicaciones en la misma frecuencia de operación.
Otro punto importante es desplazar el haz principal en un rango de $120^{\circ}$ de azimut para cubrir la zona de vigilancia.

Esto se puede realizar mecánicamente desplazando el arreglo receptor para RADAR de microondas, en cambio, ésto no es posible para RADAR de HF por las dimensiones del mismo.

Son por estas razones mencionadas (cancelación de interferencias y barrido del haz) que se deben utilizar técnicas de filtraje espacial o "beamforming" digital.

El esquema de un beamformer es el que se muestra en la figura 15 [6].
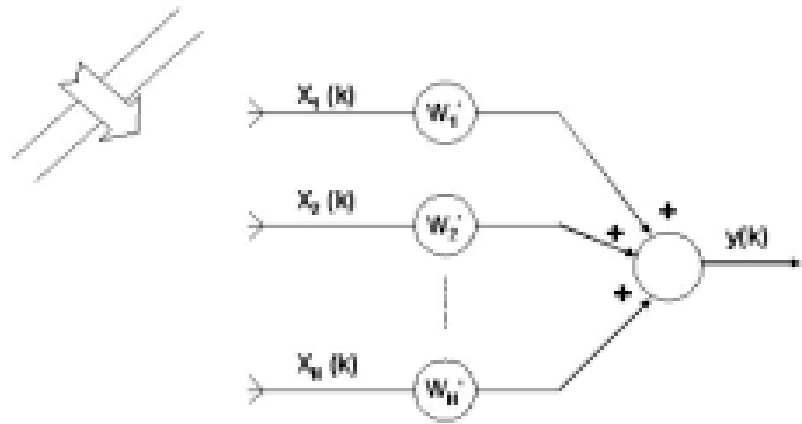

Fig. 15 Esquema de un beamformer.

La expresión de la señal de salida de un beamformer es:

$$
y(k)=w^{H} x(k)
$$

El beampattern está dado por:

$$
r(\theta, \omega)=w^{H} \cdot D(\theta, \omega)
$$

donde D es el vector de steering y está dado por:

$$
D(\theta, \omega)=\left[\begin{array}{llll}
1 & e^{j \omega \tau_{2}(\theta)} & \ldots & e^{j \omega \tau_{N}(\theta)}
\end{array}\right]^{H}
$$

donde $\tau$ son los retardos impuestos por la propagación.

La elección de los pesos "w" difieren de un algoritmo de otro. Existen variados algoritmos de beamforming para tal objetivo con ventajas y desventajas. Estos, en general, se pueden clasificar en tres grandes grupos: independiente de la data, estadísticamente óptimos y adaptivos [6].

Los algoritmos independientes de la data tratan de formar un pattern sin considerar las estadísticas de las señales entrantes al arreglo, en cambio, los estadísticamente óptimos si las consideran. Ejemplos de estos últimos se 
cuentan el MSC (Multiple Sidelobe Canceller), LCMV (Linearly Constrained Minimum Variance), GSC (Generalized Sidelobe Canceller), entre otros.

A veces, las estadísticas de las señales entrantes varían en el tiempo. En ese caso, es conveniente usar algoritmos adaptivos. Destacan el LMS (Least Mean Square) y el RLS (Recursive Mean Square). Puede ser beneficioso, en algunos casos, implementar algoritmos híbridos que combinen los 3 esquemas.

El primer algoritmo simulado fue un independiente de la data para el arreglo escogido de $24 \times 2$ (separación de $0,5 \lambda$ lineal y $0,25 \lambda$ bidimensional). En este caso clásico, se elige el vector de pesos "w" idéntico al $\mathrm{D}(\theta)$ donde " $\theta$ " es el DOA de la señal que nos interesa recibir o el ángulo de dirección del beam principal, el cual debe moverse de $30^{\circ}$ a $150^{\circ}$.

De esta forma:

$$
r=w^{H} \cdot D=D(\theta)^{H} \cdot D
$$

En este caso, la respuesta es máxima cuando el $\mathrm{D}(\theta)$ es igual al vector " $D$ " de la dirección deseada.

Los resultados de las simulaciones para haces dirigidos a $90^{\circ}, 60^{\circ}$ y $30^{\circ}$ se encuentran en las figuras 16 a la 18 . Los resultados son simétricos para $120^{\circ}$ y $150^{\circ}$.

Se logra apreciar que la supresión total del segundo lóbulo principal ocurre cuando el haz está dirigido a $90^{\circ}$ (broadside), ya que al hacer $\mathrm{w}=\mathrm{D}(\theta)$ es equivalente a multiplicar el pattern del arreglo lineal de 24 elementos con $\mathrm{d}_{\mathrm{x}}=0,5 \lambda$ por el pattern bidimensional de la figura 10 producto de una separación $\mathrm{d}_{\mathrm{y}}=0,25 \lambda$, por lo que cada vez que se mueve el haz principal, menor será la atenuación que sufrirá el segundo lóbulo principal.

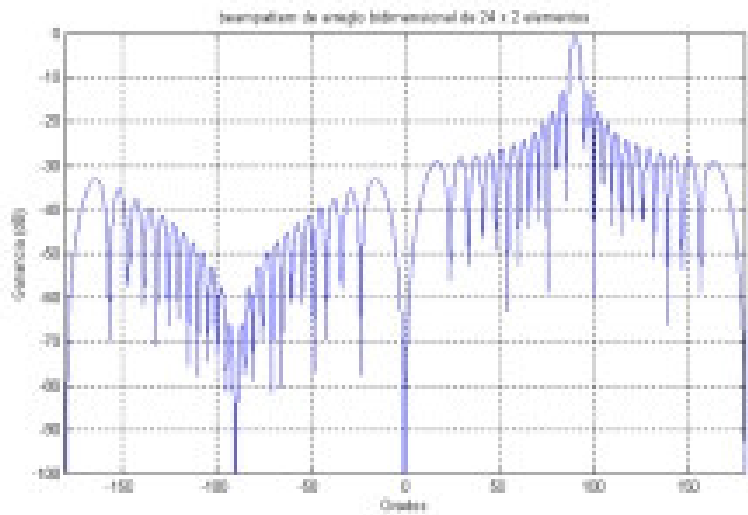

Fig. 16 Pattern azimutal dirigido a $90^{\circ}$ (broadside) del arreglo 24 x 2 usando beamforming independiente de la data.

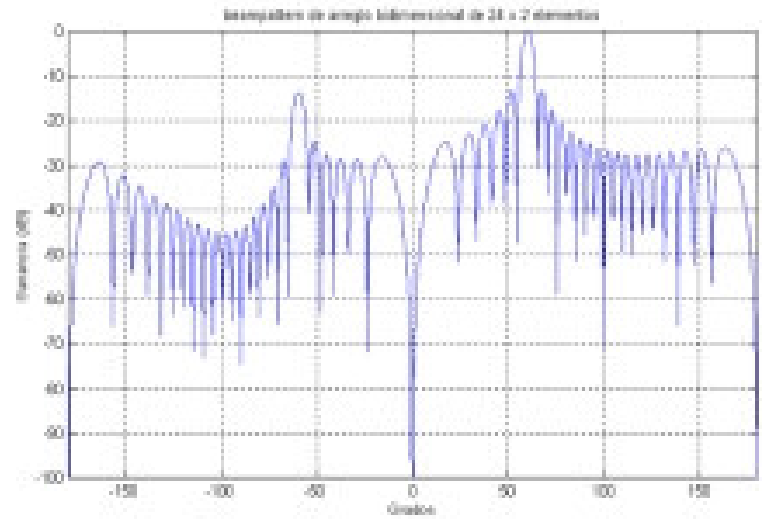

Fig. 17 Pattern azimutal dirigido a $60^{\circ}$ del arreglo $24 \times 2$ usando beamforming independiente de la data.

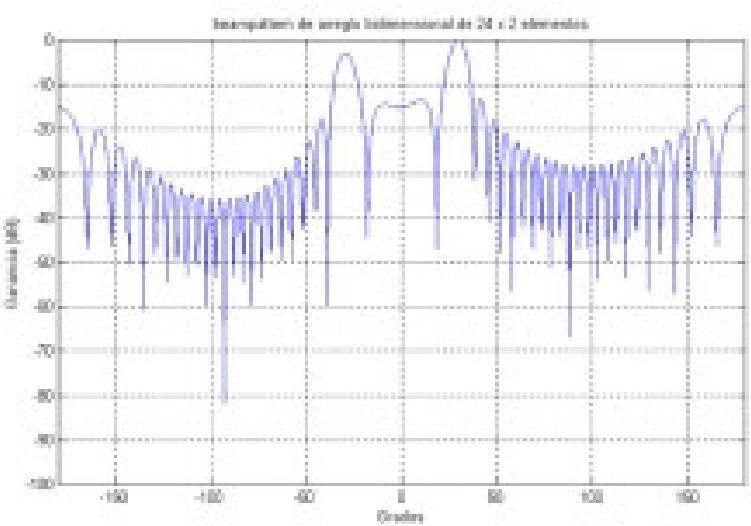

Fig. 18 Pattern azimutal dirigido a $30^{\circ}$ del arreglo $24 \mathrm{x}$ 2 usando beamforming independiente de la data.

Es evidente el peligro que involucra contar con sólo este esquema de "beamforming" para un HFSWR, debido a las posibles señales interferentes que puedan coincidir con el segundo lóbulo principal. Además, este esquema no toma en cuenta, por ejemplo, niveles de ruido existentes en el área de forma permanente o intermitente. También, los niveles de los lóbulos secundarios no son tan bajos, por lo que este esquema no se resguarda de señales interferentes que posean un nivel de potencia mucho mayor que las señales de interés. Debido a estos inconvenientes es que se implementaron esquemas de algoritmos estadísticamente óptimos, ya que éstos involucran estadísticas de las señales entrantes.

Una alternativa para eliminar completamente el segundo lóbulo principal se puede obtener con el uso de restricciones, de tal modo de forzar una baja ganancia o nulo en las direcciones de éste a través de un esquema LCMV (Linearly Constrained Minimum Variance). En este caso, los pesos "w" se obtienen de acuerdo a [6]:

$$
w=\frac{R_{x}^{-1} \cdot C}{C^{H} \cdot R_{x}^{-1} \cdot C} \cdot f
$$


Donde " $\mathrm{R}_{\mathrm{X}}$ " es la Matriz de Covarianza de las entradas, "C" es la Matriz de Restricciones y " $\mathrm{f}$ " es el Vector de Respuesta.

En este esquema LCMV, se introdujeron dos restricciones: Ganancia máxima en la dirección que se desea apuntar el haz y un Nulo en la dirección del segundo lóbulo principal. De este modo, los diagramas de radiación obtenidos son los mostrados en las figuras 19 , 20 y 21, dirigidos a los mismos ángulos del esquema anterior para apreciar las diferencias.

Es evidente la mejora de esta alternativa en relación al primer esquema de beamforming independiente de la data, puesto que en LCMV, dirigidos para los mismos ángulos de las figuras 16 a la 18, se pudo atenuar el segundo lóbulo principal. En estos momentos, nos encontramos trabajando en la implementación de algoritmos adaptivos que cubran las deficiencias de los esquemas anteriores en relación a interferencias variantes en el tiempo aplicado a HFSWR.

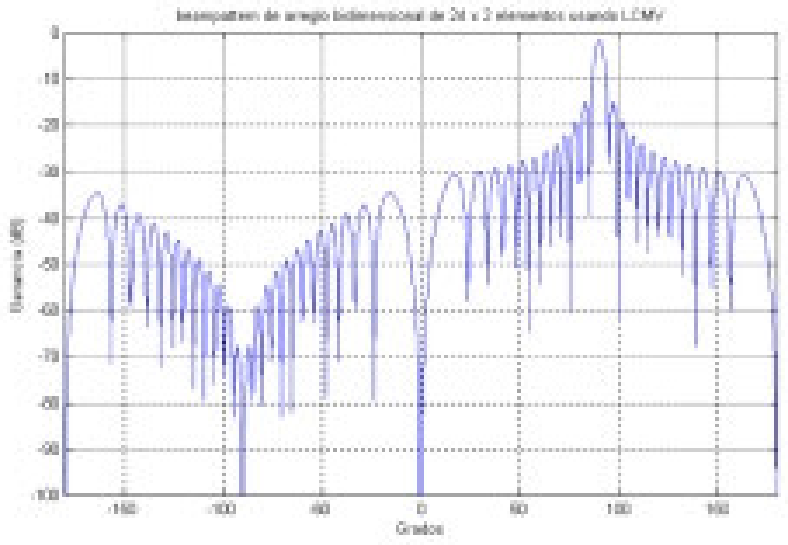

Fig. 19 Pattern azimutal dirigido a $90^{\circ}$ (broadside) del arreglo $24 \times 2$ usando LCMV.

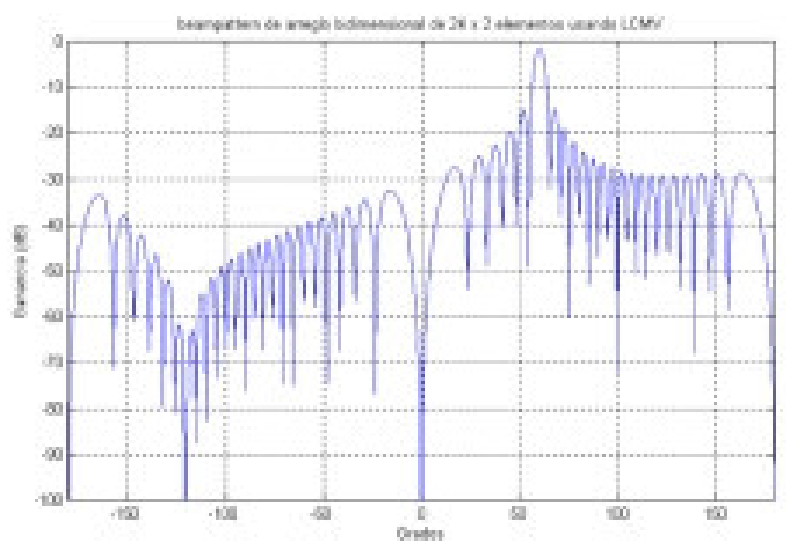

Fig. 20 Pattern azimutal dirigido a $60^{\circ}$ del arreglo $24 \times 2$ usando LCMV.

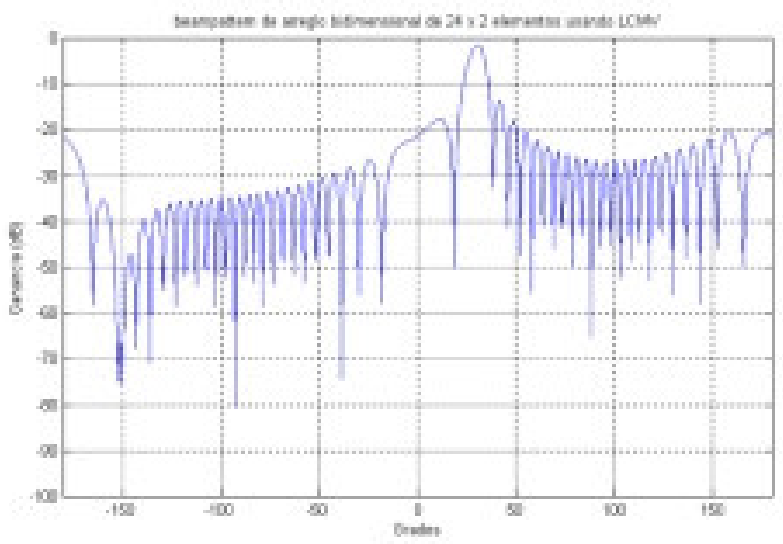

Fig. 21 Pattern azimutal dirigido a $30^{\circ}$ del arreglo $24 \times 2$ usando LCMV.

\section{CONCLUSIONES}

Se acaban de revisar los conceptos fundamentales de un HFSWR y las variables que lo afectan en su rendimiento, notando que tiene diferencias importantes con los del tipo que trabajan en la banda de microondas. Para el diseño de cualquiera de sus partes se deben tener en cuenta parámetros como el ruido, interferencia, reflectividad, propagación de HF en superficies rugosas (mar), plano de tierra, arreglo transmisor, receptor y muchos más.

Queda claro, con esta revisión de las propiedades de HFSWR, que la vigilancia de la ZEE de nuestro territorio nacional se vería bastante beneficiada con este tipo de sensores debido, principalmente, a que se trata de un sistema de vigilancia en tiempo real y su alcance alcanza las $200 \mathrm{MN}$.

Se analizaron arreglos de antenas para su utilización en el sistema receptor de un HFSWR, llegando a la elección de un arreglo de $24 \times 2$ con separaciones $d_{X}=0,5 \cdot \lambda$ y $\mathrm{d}_{\mathrm{Y}}=0,25 \cdot \lambda$. Se analizaron algunos esquemas de beamforming para desplazar, dirigir el haz y eliminar el segundo lóbulo principal, y poder cumplir la tarea del barrido en un RADAR de este tipo.

\section{AGRADECIMIENTOS}

Agradecemos a la Dirección de Investigación y Postgrado de la PUCV por el apoyo al proyecto DI 204.705-2004. 


\section{REFERENCIAS}

[1] S. Anderson, B. Bates, M. Tyler. "HF Surface wave Radar and its role in littoral warfare". Journal of Battlefield Technology. Vol. 2. $\mathrm{N}^{\mathrm{o}}$ 3. November. 1999.

[2] L. Sevgi. “An Integrated Maritime Surveillance System Based on High-Frecuency Surface-Wave Radars, Part 1: Theorical Background and Numerical Simulations". IEEE Antennas and Propagation Magazine. Vol. 43. $\mathrm{N}^{\circ}$ 4. August. 2001.
[3] M. Skolnik. "Radar Handbook". Mc Graw-Hill. 1990.

[4] C. Balanis. "Antenna Theory: Analysis and Design”. John Wiley \& Sons. Second Edition. USA. 1997.

[5] B. Van Been. "Beamforming: A Versatile Approach to Spatial Filtering". IEEE ASSP MAGAZINE. April. 1998. 